In Cres. Vol. $4 N^{\circ}$ 2: pp. 257-267, 2013

\title{
ESTUDIO COMPARADO DEL DISEÑO DEL ÁREA DE INVESTIGACIÓN EN LA LICENCIATURA EN PEDAGOGÍA*
}

\author{
COMPARATIVE STUDY IN THE RESEARCH AREA \\ AT THE DEGREE IN PEDAGOGY
}

\begin{abstract}
Mónica Guadalupe Tovar Gutiérrez', María Guadalupe Veytia Bucheli ${ }^{2}$
\end{abstract}

\begin{abstract}
RESUMEN
El estudio que se expone en este artículo es de tipo exploratorio descriptivo, el cual presenta las tendencias que caracterizaron el Diseño Curricular de los programas de Licenciatura en Pedagogía en relación con el desarrollo del área de investigación en la década del 2000 al 2010. Se tomaron como referentes universidades de reconocido prestigio que ofertan la carrera de Pedagogía en México, Chile, Cuba y España, al considerarse el desarrollo que han alcanzado estos países en materia educativa. La investigación incluye el análisis de los planes de estudio en cuanto a sus objetivos, perfiles de ingreso y egreso, con la intención de identificar las posiciones asumidas por universidades de diferentes partes del mundo sobre el desarrollo de la investigación que generan sus estudiantes para obtener el título de Licenciado en Pedagogía, lo que permitió identificar puntos de coincidencia y diferencia entre ellos, así como reflexionar sobre el perfil que se requiere para el pedagogo del siglo XXI. Este trabajo se realizó por medio de matrices que permitieron comparar estos elementos de análisis.

PALABRAS ClAVE: Diseño curricular, Estudiantes, Investigación, Pedagogía.
\end{abstract}

* Recibido: 15 de mayo del 2013; aprobado: 10 de octubre del 2013.

1 Doctora en Educación. Maestra en Docencia Superior. Especialista en Docencia Superior. Licenciatura en Educación. Catedrática de la Unidad de Posgrado de la Universidad Tangamanga.

2 Doctora en Gestión Educativa. Maestra en Educación. Especialista en Entornos Virtuales de Aprendizaje. Licenciada en Educación. Catedrática de la Unidad de Posgrado de la Universidad Tangamanga. 


\begin{abstract}
The study discussed in this article is an exploratory, which presents trends that characterized the Curriculum Design Degree in Education programs relating to the development of the research area in the decade from 2000 to 2010, were taken as concerning prestigious universities that offer the teaching career in Mexico, Chile, Cuba and Spain, when considering the development that these countries have achieved in education. The research was carried out, including the analysis of the curriculum in terms of its objectives, entry and exit profiles, with the intention of identifying the positions taken by universities from around the world on the development of research generate their students for the degree of Bachelor of Education, which allowed us to identify points of agreement and difference between them, and reflect on the profile required for the XXI Century Educator. This work was carried out by means of the realization of arrays which allowed analysis comparing these elements.
\end{abstract}

KEY WORDS: Curriculum Design, Students, Research, Education.

\title{
INTRODUCCIÓN
}

La educación superior en la actualidad enfrenta retos que le demanda la sociedad del siglo XXI, en la cual se requiere un proceso educativo congruente con las necesidades de la sociedad; por lo tanto, surge la necesidad de adecuar $\mathrm{o}$ innovar el currículum como un componente medular de la acción educativa en cada institución.

De acuerdo a Miyajira (2009), la formación en la educación superior es esencial para crear la capacidad intelectual de producir y utilizar conocimientos, así como para el aprendizaje permanente, pues ya no solo es suficiente un aprendizaje para la vida, sino durante toda la vida, lo cual constituye un reto para la universidad.

La UNESCO (1998) menciona como primera misión fundamental en la educación superior la de educar, formar y realizar investigaciones en torno de la capacitación de individuos calificados en el campo laboral, el aprendizaje permanente y la participación activa de la sociedad; y a su vez, se basa en torno a tres pilares que son la docencia, la investigación y la extensión.

La investigación universitaria, de acuerdo a Restrepo (2002, pág. 196), es "un proceso de búsqueda de nuevo conocimiento, proceso caracterizado por la creación del acto, por la innovación de ideas, por los métodos rigurosos utilizados, por la autocrítica y por la validación y juicio de pares. A la investigación está unida íntimamente la creatividad, ya que en buena medida, los resultados de la investigación son también creación de conocimiento o de tecnología”. 
Esta postura se vincula con las características de la sociedad del siglo XXI, marcadas por la complejidad, lo global y lo local, es indispensable que la educación superior modifique sus paradigmas transitando de una educación tradicional a otra que permita la generación y aplicación del conocimiento a través de nuevas propuestas; en este sentido, si se habla del estudiante de la licenciatura en pedagogía, es pertinente que sus programas consideren, tanto una formación docente como una formación en investigación, ya que constituyen dos pilares claves para elaborar un diagnóstico sobre el contexto educativo, así como para utilizar las herramientas que permitan llevar a cabo propuestas de mejora e intervención (Guerrero, 2007).

En México, el Plan Nacional de Desarrollo 2006-2012 (pág. 196) precisa la trascendencia de la educación superior, donde se señala dentro de sus procesos fundamentales convertir a este nivel educativo en motor para alcanzar mejores niveles de vida, con la capacidad de transmitir, generar y aplicar conocimientos; para, de esta manera, lograr la inserción en la emergente economía del conocimiento; sin embargo "la educación superior que se imparte en el país ocupa el lugar 15 de las 33 naciones de América Latina y el Caribe que fueron evaluadas por el Instituto de Estadística de la Organización de las Naciones Unidas para la Educación, la Ciencia y la Cultura (UNESCO).

La consolidación de este nivel educativo como un sistema de mayor cobertura, más abierto, diversificado, flexible, articulado y de alta calidad, es esencial para el desarrollo del país. Por lo tanto, en este trabajo se plantea un eje fundamental que lo constituye la articulación entre currículum e investigación, como una respuesta a los estudiantes universitarios.

Desde la postura de Stenhouse (2003), se determina el currículo como una prescripción por escrito de lo que se pretende realizar en las escuelas, lo cual debe permitir vincularlo con la realidad de la universidad, el aula y las exigencias sociales. El currículum es aquello que acontece a los estudiantes en la escuela como resultado del trabajo de los docentes; incluye todas las experiencias de los alumnos cuya responsabilidad asume la escuela.

Gimeno (2007, pág. 65) precisa que "el currículum suele reflejar un proyecto educativo globalizador, que agrupa diversas facetas de la cultura, el desarrollo personal y social; de las necesidades vitales de los individuos para desenvolverse en sociedad, destrezas y habilidades consideradas fundamentales".

Esto lleva a conceptualizar el currículum como una serie de saberes conceptuales, procedimentales y actitudinales que requieren ser utilizados con efi- 
cacia y eficiencia en un contexto específico; en este caso, se habla del contexto laboral en el cual se desempeñan los egresados.

Las prácticas educativas en la sociedad del conocimiento son dinámicas, por lo que "los diseñadores y elaboradores del currículum enfrentan el desafío de identificar aquellos conocimientos básicos, fundantes, estructurantes, que le permiten a la persona seguir aprendiendo autónomamente" (Magendzo, 2008, pág. 208) y de esta manera, en su diseño el bloque investigativo es una exigencia, pues permite establecer un vínculo entre el carácter científico de la educación con los valores y el compromiso social, desde una postura ética, reflexiva y crítica.

En el presente trabajo se precisa la importancia que las universidades, desde su papel educativo, deben formar investigadores en correspondencia con las tendencias del desarrollo social y educativo, así como de las necesidades contextuales, rompiendo con la brecha que aún existe entre el mundo de la escuela, por un lado, y el de la sociedad, el trabajo y el desarrollo, por otra.

El componente investigativo en los planes de estudio a nivel universitario requiere ser revisado y, en especial, en la licenciatura en pedagogía, la cual vincula la docencia con la investigación para la generación de cambios significativos en el contexto; sin embargo, no siempre se considera, por lo que surge el problema científico: ¿En qué elementos del diseño curricular se hace evidente el componente investigativo en la Licenciatura en Pedagogía que ofertan universidades nacionales e internacionales?

\section{OBJETIVO GENERAL}

Valorar las características del diseño curricular del área de investigación en la licenciatura en pedagogía que ofertan universidades nacionales e internacionales.

\section{OBJETIVOS ESPECÍFICOS}

a) Identificar las semejanzas y diferencias entre el diseño curricular de la licenciatura en pedagogía que se oferta en diferentes partes del mundo; y

b) Analizar en qué elementos del diseño curricular de la licenciatura en pedagogía está presente el componente investigativo.

La importancia de la investigación reside en que a partir del análisis del diseño curricular de la licenciatura en pedagogía que ofertan universidades nacionales e internacionales, se podrá identificar las fortalezas y áreas de oportunidad de manera global, así como tener las bases para que, en una segunda 
etapa, se diseñe una propuesta para enriquecer el componente investigativo de la licenciatura en pedagogía que ofrece la Universidad Tangamanga, tanto en sus programas de esta área, como de manera transversal, en las diferentes asignaturas que la conforman.

\section{MATERIALES Y MÉTODOS}

La investigación es exploratoria y con un enfoque cualitativo, a través de la valoración de la vinculación de la investigación en los planes de estudio de las licenciatura en pedagogía tanto a nivel nacional como internacional a partir de los siguientes indicadores: objetivo de la carrera, perfil de egreso, competencias que tributa, materias en el bloque metodológico, análisis de programas, así como formas de titulación.

Para el desarrollo de esta investigación se seleccionaron 13 instituciones de educación superior que ofertan la licenciatura en pedagogía. De estas 8 son universidades nacionales reconocidas, así como 5 universidades internacionales de España, Cuba y Chile.

El análisis de la información obtenida se trabajó a partir de la elaboración de una matriz de doble entrada con los indicadores y las universidades referidas, lo cual permitió identificar semejanzas y diferencias entre las diferentes instituciones, así como fortalezas y áreas de oportunidad. A su vez, se identificaron tendencias y características sobresalientes, realizando una valoración del significado que se otorga a la investigación que se implementa en las universidades y otras instituciones de educación superior donde se forman docentes.

El análisis incluye el estudio de las competencias propuestas en el área de la educación en el Proyecto Tuning en América Latina (2004-2008), así como la revisión de investigaciones realizadas como parte del proyecto.

\section{RESULTADOS}

Los principales resultados que se obtienen del análisis del Diseño Curricular de la Licenciatura en Pedagogía se presentan a continuación:

\section{REFERENTE A LOS OBJETIVOS DE LA FORMACIÓN}

El objetivo de los planes de estudio declara las características fundamentales que se proponen en la formación; por lo que se consideró importante apreciar en qué medida en este se destaca la investigación. 
En el $69 \%$ de los planes de estudio analizados se destaca la investigación relacionada con el objetivo a alcanzar. La mayoría opta por la competencia a alcanzar en la formación. En las universidades nacionales se enfatizó la presencia de la investigación en los planes de la Universidad Autónoma; a la vez, llama la atención que en los planes de las escuelas normales no se considera la investigación en los objetivos de la formación.

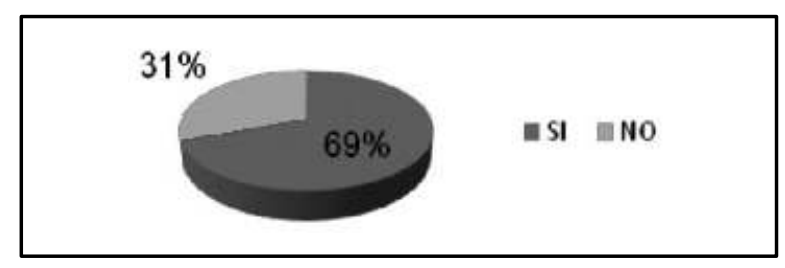

Gráfico 1. Universidades que destacan la investigación en los objetivos de la formación. Fuente: Autor.

La idea de la investigación en los objetivos de los planes trasciende con los planteamientos de Stenhouse (2003, pág. 178) cuando menciona "propongo que exigir una enseñanza basada en la investigación es pedirnos a nosotros, como profesores, que compartamos con nuestros alumnos o estudiantes nuestro proceso de aprendizaje de la sabiduría que no poseemos, de manera que ellos puedan poner en una perspectiva critica el aprendizaje que nosotros confiamos en que es nuestro".

\section{REFERENTE AL PERFIL DE EGRESO}

Otro de los aspectos estudiados es el perfil de egreso, en el que se identificó su vinculación con los referentes a la formación de investigación. El análisis realizado arrojó que un $69 \%$ de las universidades promueven en el profesionista competencias para la investigación; el $31 \%$ no señalan un perfil encaminado a la investigación.

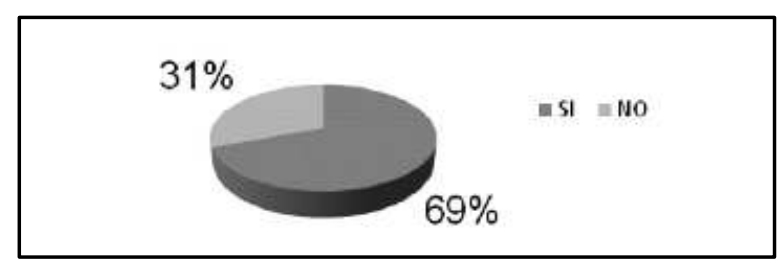

Gráfico 2. Presencia de la Investigación en el

Perfil de Egreso. Fuente: Autor. 
Las referencias en el perfil se refieren al desarrollo de competencias de investigación, lo que relacionan con la solución de problemas educativos, realización de proyectos, implementación de innovaciones didácticas, estrategias, principalmente. En el perfil se aprecia un enfoque, tanto hacia la investigación cualitativa como a la cuantitativa. Destacan un enfoque mixto en la Universidad Autónoma en la Facultad de Filosofía y Letras, así como las universidades de Salamanca (España) y el Instituto Enrique José Varona, de Cuba.

\section{REFERENTE A LAS MATERIAS DEL ÁREA DE INVESTIGACIÓN}

En el aspecto de inclusión de disciplinas en la línea de investigación en los planes de estudio analizados, destacan materias como Metodología de la Investigación, Seminario de Tesis, Investigación Educativa, entre otras denominaciones. Se pone de manifiesto que el $38 \%$ de las universidades incluyen entre 5 a 7 materias hacia la investigación, el $23 \%$ implementa 3 materias, el $31 \%$ incluye 2 materias y el $8 \%$ no imparte materias de investigación.

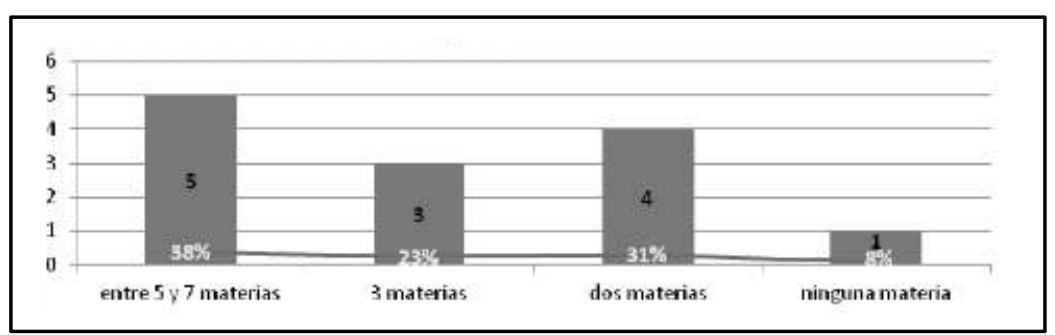

Gráfico 3. Materias de Investigación en el Plan de Estudios.

Fuente: Autor.

Se resume que en las universidades analizadas un $91 \%$ implementa en sus contenidos la formación en la investigación, lo que se refleja en la inclusión de objetivos, hechos, conceptos, actitudes, entre otros componentes, para que sean logrados por los alumnos en su formación profesional. Se aprecia que consideran en los programas de las materias la investigación los diferentes componentes característicos del contenido de aprendizaje de esta área.

\section{REFERENTE AL TIPO DE ACTIVIDADES DE INVESTIGACIÓN QUE SE PROPONEN}

Otra dimensión relevante es el tipo de actividades relacionadas con la investigación que se incluyen en los planes de estudio. Del total de 13 progra- 
mas revisados, en 9 aparecen referencias al tipo de actividad que se exige a los estudiantes en la formación en la investigación. El 89\% de las universidades refieren exigir entre las tareas el diseño de investigación, sólo una universidad no lo refiere (Universidad de Aragón), cuyo modelo de investigación es cualitativo. Si bien la mayoría considera la recolección y su análisis, sólo una de cada cuatro incluye el análisis estadístico. La exigencia de informe de investigación aparece en la mayoría de los documentos revisados de todas las universidades; es posible que en las que no se refieren puedan exigirlo.

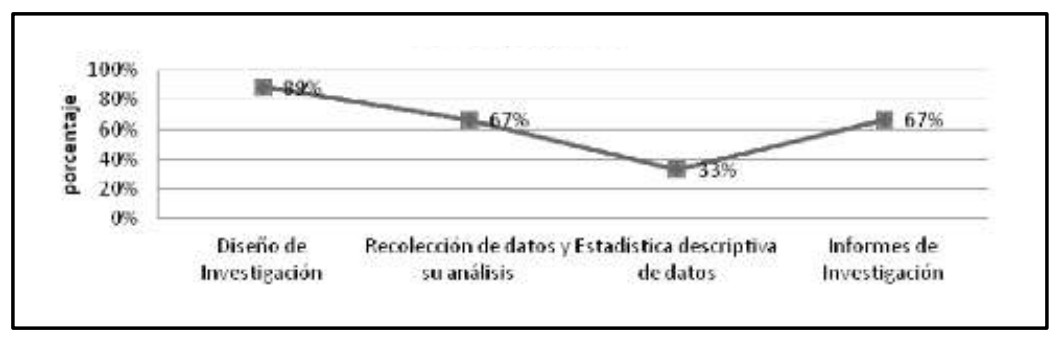

Gráfico 4. Actividades de Investigación que se incluyen en el Diseño de los Programas. Fuente: Autor.

\section{TIPOS DE INVESTIGACIÓN QUE SE PROPONEN EN LAS UNIVERSIDADES ESTUDIADAS}

La información revisada en las diferentes universidades pone de manifiesto la tendencia en el área de la educación a privilegiar la investigación de corte cualitativo en el nivel de licenciatura en un $67 \%$, por sobre el cuantitativo. Destaca que el enfoque mixto de investigación sólo se refiere en dos instituciones de las analizadas: la Universidad de Salamanca y el Instituto Enrique J. Varona, de Cuba, siendo esta una posición más actual.

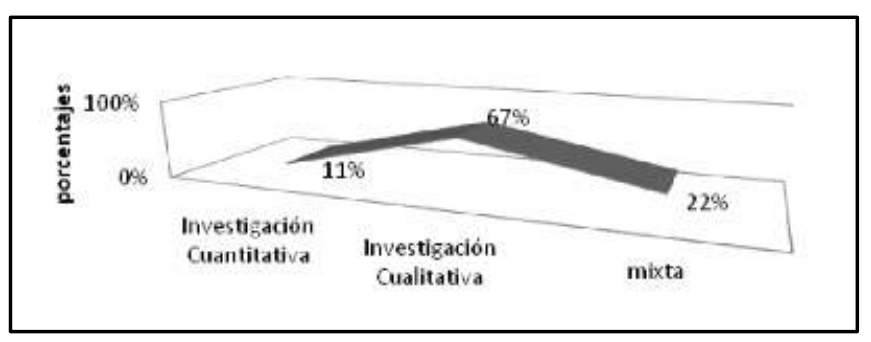

Gráfico 5. Tipos de Investigación.

Fuente: Autor. 


\section{LA TITULACIÓN Y SU RELACIÓN CON LA DEFENSA DE TRABAJOS DE INVESTIGACIÓN}

Los resultados son que el $62 \%$ tesis, el $38 \%$ tesina, $8 \%$ actividades de investigación, $8 \%$ informe académico, $15 \%$ informe por servicio social, $8 \%$ memoria de desempeño, $8 \%$ postgrado, $8 \%$ CENEVAL, $8 \%$ reporte de experiencia, $8 \%$ proyecto de innovación docente, $8 \%$ propuesta pedagógica, $8 \%$ proyecto de desarrollo educativo, $8 \%$ monografía, $8 \%$ trabajos experimentales, $8 \%$ trabajos de investigación.

Esto indica que las universidades promueven diversas formas de titulación; pero con el propósito de aprender a investigar se priorizan la tesis y la tesina.

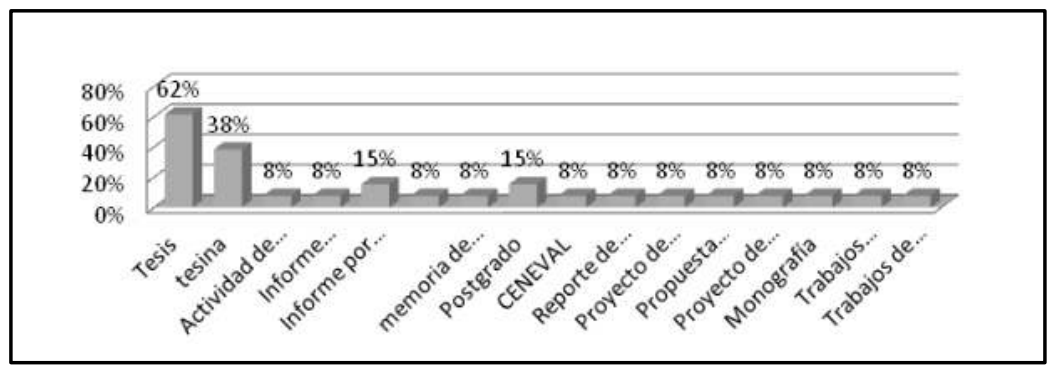

Gráfico 6. Tipo de Titulación.

Fuente: Autor.

La propuesta de titulación por tesis y tesina arrojan porcentajes de $62 \%$ por tesis y $38 \%$ por tesina, permitiendo vincular el proceso educativo con la investigación, favoreciendo experiencias de aprendizaje que propician actitudes científicas de los profesionistas que egresan.

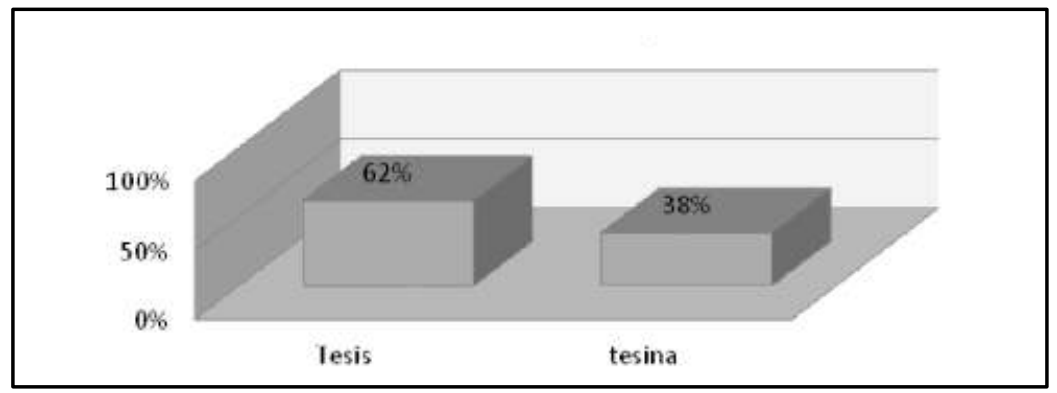

Gráfico 7. Titulación con Tesis y Tesina.

Fuente: Autor. 
En este trabajo se plantea que en el contexto educativo actual, las universidades estudiadas se caracterizan por enfocar sus procesos educativos desde un carácter científico, considerando la investigación como una función profesional del pedagogo contemporáneo, permitiéndole preparase para buscar soluciones científicas a los problemas del quehacer profesional.

\section{DISCUSIÓN}

A partir del análisis realizado, se encuentra la presencia del bloque metodológico en el diseño curricular del Plan de Estudios de la Licenciatura en Pedagogía ofertada por diferentes instituciones tanto a nivel nacional como internacional, en los elementos como son: objetivos, perfil de egreso, materias relacionadas con la investigación, tipo de actividades que se incluyen en el programa referentes a la investigación, y las formas de titulación.

Entre los resultados que obtienen porcentajes más altos en cuanto a la presencia de investigación, son los que se refieren a los objetivos y perfil de egreso con un $69 \%$, el $38 \%$ lo obtienen los planes de estudio que manejan entre 5 a 7 relacionadas con la investigación; el porcentaje más alto de actividades para desarrollar la investigación se presenta en el momento de diseñarla con un $89 \%$; el tipo de investigación con porcentaje más alto se refiere a la investigación cualitativa con un $67 \%$; y por último, sobre las 16 opciones de titulación, la tesis maneja el mayor porcentaje con un $62 \%$.

\section{CONCLUSIONES}

El estudio exploratorio que se llevó a cabo para valorar las características del diseño curricular de los programas de licenciatura en pedagogía ofertados en diferentes universidades nacionales e internacionales, permitió establecer comparaciones en el diseño en cuanto a los objetivos, perfiles de egreso, materias y actividades relacionadas con la investigación y formas de titulación, obteniendo porcentajes altos en cuanto a la presencia de este componente en el diseño curricular.

Este primer análisis permite identificar fortalezas, como el número de materias en las que se trabaja la investigación en los diferentes planes de estudio de la licenciatura en pedagogía, y las actividades para desarrollar el diseño de su investigación, así como áreas de oportunidad como es el inicio de la investigación, ya que varias universidades lo hacen en forma tardía en sus últimos semestres o cuatrimestres. 
Estudio comparado del diseño del área de investigación en la licenciatura en pedagogía

\section{REFERENCIAS BIBLIOGRÁFICAS}

Gimeno SACRISTÁN, J. (2007) El currículum: una reflexión sobre la práctica. España: Ed. Morata.

GUERRERo USEDA, M.E. (2007) Formación de habilidades para la investigación desde el Pregrado. Recuperado de: http://www.scielo.org.co/pdf/acp/v10n2/v10n2a18.pdf

MAGENDKO, A. (2008) Dilemas del currículum y la Pedagogía. Analizando la Reforma Curricular desde una perspectiva crítica. Santiago de Chile. LOM Ediciones.

MiYAHIRA, j. (2009) La investigación formativa y la formación para la investigación en el pregrado. Recuperado de: http://www.scielo.org.pe/pdf/rmh/v20n3/v20n3e1.pdf

PLAN NACIONAL DE DESARROLlo. Recuperado de: http://pnd.calderon.presidencia.gob.mx/ igualdad-de-oportunidades/transformacion-educativa.html

RESTREPO, B. (2002) Investigación formativa e investigación productiva de conocimiento en la Universidad. Revista Nómadas.

STENHOUSE L. (2003) Investigación y Desarrollo del Currículum. Madrid. Ed. Morata.

TUNNING AMÉRICA LATINA (2004- 2008) Recuperado de: http://tuning.unideusto.org/tuningal/

UNESCO (1999) Declaración mundial sobre la Educación Superior en el Siglo XXI: Visión y Acción. Recuperado de: http://www.unesco.org/education/educprog/wche/declaration_spa.htm

Correspondencia con: María Guadalupe Veytia Bucheli dra.veytiabucheli@gmail.com 